\title{
Thermal Particle Production in Two Taub-Nut Type Spacetimes
}

\author{
Alan S. Lapedes \\ D.A.M.T.P., University of Cambridge, Cambridge CB3 9EW, England
}

\begin{abstract}
The Hartle-Hawking method of deriving black hole radiance (the "Hawking Process") has been extended to non-asymptotically flat de Sitter spacetime by Gibbons and Hawking. They derive a thermal spectrum of radiation detectable by suitable observers. We extend this work to Taub-Nut spacetime and a related and more physical spacetime constructed from it by Siklos by complex analytic continuation and unwrapping. Suitable observers are found to detect thermal spectra in these two spacetimes as well.
\end{abstract}

\section{Introduction}

In this paper particle production in two related homogeneous and anisotropic spacetimes is considered. We use the framework for calculating particle production in non-asymptotically flat spacetime developed by Gibbons and Hawking [1] for de Sitter space as an extension of the Hartle-Hawking [2] path integral method of deriving black hole radiance. The first spacetime we will consider is Taub-Nut space which is not only non asymptotically flat (topology $R \times S^{3}$ ) but is non Hausdorff (however without bifurcating geodesics), has closed timelike and null lines and suffers from geodesic incompleteness. In this spacetime a suitable observer (unfortunately one moving on a closed time-like line) would detect a thermal spectrum, however it is truncated at a low fundamental frequency and is discrete in integer multiples of this fundamental frequency. We consider this pathological spacetime mainly as an illustration of the elegance and power of the path integral method.

The second more physical spacetime considered is a Bianchi-type VIII spacetime constructed by Siklos [3] from Taub-Nut space by complex analytic continuation and unwrapping. The resultant manifold is Hausdorff, and although there still exist closed time-like lines in this Type VIII Taub-Nut space we choose an observer who does not move on one. This observer is also found to observe a thermal spectrum.

The paper is divided into five sections. In Section 1 we review the global properties of Taub-Nut space. In Section 2 we review the Hartle-Hawking path 
integral method and use it to derive the truncated thermal spectrum mentioned above. In Section 3 we explain Siklos' construction of the Type VIII Taub-Nut space and in Section 4 we derive the thermal spectrum seen by a suitable observer in this spacetime. Section 5 lists the conclusions.

\section{Taub-Nut-Space: The Global Properties}

Taub-Nut space refers to the extension of the original Taub universe [4] which is a spatially homogenous, anisotropic, vacuum solution of Einstein's equations with topology $R \times S^{3}$. In a coordinated basis the metric is given by:

$$
d s^{2}=-U^{-1} d t^{2}+(2 l)^{2} U(d \psi+\cos \theta d \phi)^{2}+\left(t^{2}+l^{2}\right)\left(\sin ^{2} \theta d \phi^{2}+d \theta^{2}\right)
$$

where:

$$
U(t)=-1+\frac{2\left(m t+l^{2}\right)}{t^{2}+l^{2}}
$$

and $m, l$ are positive constants, and $\theta, \phi, \psi$ are Euler angle coordinates on $S^{3}$ :

$0 \leqq \psi \leqq 4 \pi, \quad 0 \leqq \theta \leqq \pi, \quad 0 \leqq \phi \leqq 2 \pi$.

The metric is singular when $U=0$ at $t=t_{ \pm}=m \pm\left(m^{2}+l^{2}\right)^{1 / 2}$, however there exist extensions across these coordinate singularities, somewhat analogous to the Finkelstein-type extensions of the Reisner-Nordstrom solution across $r=r_{ \pm}$, that yield the space discovered by Newman, Unti and Tamburino [5] (Nut space). The $t=t_{ \pm}$surfaces are horizons analogous to the $r=r_{ \pm}$surfaces in the Reisner-Nordstrom solution.

One extension is defined by:

$$
v=\psi+t / 2 l+\frac{1}{2 \kappa_{+}} \ln \left(t-t_{+}\right)+\frac{1}{2 \kappa_{-}} \ln \left(t-t_{-}\right), \quad \kappa_{ \pm}=\frac{ \pm l\left(t_{+}-t_{-}\right)}{\left(t_{ \pm}\right)^{2}+l^{2}}
$$

which results in a metric analytic over the full range $-\infty<t<\infty$. The region $t_{-}<t<t_{+}$is isometric with the original Taub space. Another inequivalent analytic extension is defined by:

$$
u=\psi-t / 2 l-\frac{1}{2 \kappa_{+}} \ln \left(t-t_{+}\right)-\frac{1}{2 \kappa_{-}} \ln \left(t-t_{-}\right),
$$

which again results in an analytic metric over the full range $-\infty<t<+\infty$. The region $t_{-}<t<t_{+}$is isometric with the original Taub space. Each extension has closed time-like lines in the Nut regions for $t<t_{-}$and for $t>t_{+}$. There also exist incomplete geodesics.

The full extension analogous to the Kruskal-type extension of the ReisnerNordstrom solution is complicated by technical difficulties and is clearly described by Hawking and Ellis [6]. Requiring the global extension to be a manifold results in the elimination of two points $p_{+}, p_{-}$in Figure 1 . There are incomplete geodesics that hit these deleted points, however the spacetime already contained incomplete geodesics so this is of little consequence. In addition the global extension is nonHausdorff, however this does not imply bifurcating geodesics in this case and is a 


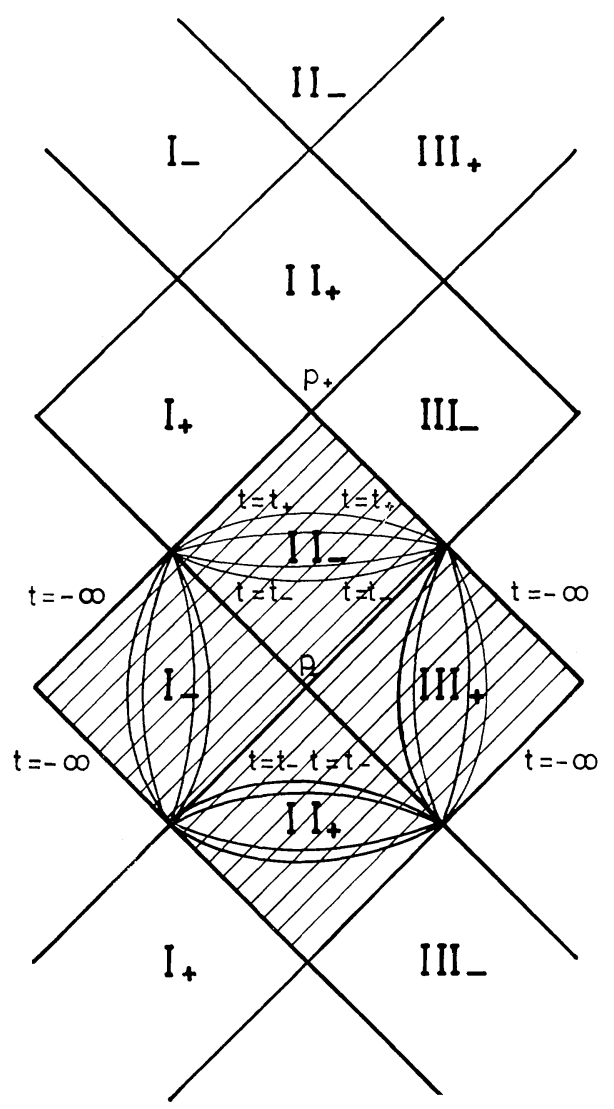

Fig. 1. The maximally extended covering space of a two dimensional section of Taub-Nut space. Regions $\mathrm{II} \pm$ are Taub universes while regions $\mathrm{I} \pm \mathrm{III} \pm$ are Nut regions. Homogeneous surfaces in the Nut region are the timelike $t=$ const surfaces. The Nut region contains closed timelike lines. Points $p_{+}$and $p_{-}$are deleted

mere technicality. Since we need a coordinate chart sufficient to cover the $t=t_{-}$ horizon (or $t=t_{+}$, but not both simultaneously) we consider the following coordinate transformation:

$$
\begin{aligned}
\bar{U}_{ \pm} & =-e^{-\left(\kappa_{ \pm}\right) u} \\
\bar{V}_{ \pm} & =e^{\left(\kappa_{ \pm}\right) v}
\end{aligned}
$$

so that:

$$
\begin{aligned}
d s^{2}= & \frac{-4 l^{2}}{\kappa_{ \pm}^{2}} \frac{\left(t-t_{-}\right)\left(t-t_{+}\right)^{-\kappa_{ \pm} t / l}}{\left(t-t_{-}\right)^{\kappa_{ \pm} / \kappa_{-}}\left(t-t_{+}\right)^{\kappa_{ \pm} / \kappa_{+}}} \frac{d \bar{U}_{ \pm} d \bar{V}_{ \pm}}{\left(t^{2}+l^{2}\right)} \\
& +4 l^{2} v(t)\left[\frac{1}{\kappa_{ \pm}} \cos \theta\left(d \bar{V}_{ \pm}-d \bar{U}_{ \pm}\right) d \phi+\cos ^{2} \theta d \phi^{2}\right]+\left(t^{2}+l^{2}\right) d \Omega^{2} .
\end{aligned}
$$


One can write $\bar{V}_{ \pm}=T_{ \pm}+X_{ \pm}, \bar{U}_{ \pm}=T_{ \pm}-X_{ \pm}$to obtain a non null form. The metric in the $\bar{U}_{ \pm}, \bar{V}_{ \pm}$coordinates is analytic everywhere except at $t=t_{\mp}$. The Penrose diagram of the maximally extended covering space of a two dimensional section of Taub-Nut space is shown in Figure 1. We will use a chart analytic at $t=t_{-}$which covers the shaded region in Figure 1.

\section{The Hartle-Hawking Method: Taub-Nut Space}

To derive the thermal spectrum seen by an observer in the NUT region moving on a $t=$ constant (closed) time-like line it is only necessary to consider the analyticity of the Feynman propogator. We will follow a procedure similar to that used by Hartle and Hawking [2] and Gibbons and Hawking [1] in computing the thermal spectrum seen by appropriate observers in the black hole and de Sitter cases respectively. In this method the amplitude $K\left(x, x^{\prime}\right)$ for a particle to propagate from one spacetime point $x^{\prime}$ to another $x$, is expressed as an integral over all paths connecting the two points:

$$
K\left(x, x^{\prime}\right)=\sum_{\text {paths }} e^{i S\left(x, x^{\prime}\right) / \hbar} \delta[x],
$$

where $S\left(x, x^{\prime}\right)$ is the classical action for a particular path connecting $x^{\prime}$ and $x$, and $\delta[x]$ is a suitable measure over the space of paths. The path of the particle is parameterized by giving the four space time coordinates $x^{\alpha}$ as functions of a parameter time $w$, and the action functional is then chosen to be:

$$
S[x(w)]=\frac{1}{4} \int_{0}^{w} d w g(\dot{x}, \dot{x}),
$$

where $x$ stands for all four coordinates and $x(0)=x^{\prime}, x(W)=x$. Here $g$ is the metric on the curved spacetime and $\dot{x}$ is the tangent vector with components $d x^{\alpha} / d w$.

An integral representation for the propagator $K\left(x, x^{\prime}\right)$ is found by considering the quantity:

$$
F\left(W, x, x^{\prime}\right)=\int \delta[x(w)] e^{\frac{i}{4} \int_{0}^{W} g(\dot{x}, \dot{x}) d w} .
$$

This is the amplitude that an observation of a spacetime position at one parameter time yields the value $x^{\prime}$ and a second observation a parameter time $W$ later yields the value $x$. This integral is a functional integral over all paths connecting $x(0)=x^{\prime}$ and $x(W)=X$. The propagator $K\left(x, x^{\prime}\right)$ is constructed from $F(W, x, \dot{x})$ by summing over all $W$ with the appropriate weighting factor: $i \exp \left(-i m^{2} W\right)$. The expression for the propagator then takes the integral form:

$$
K\left(x, x^{\prime}\right)=i \int_{0}^{\infty} d W \exp \left(-i m^{2} W\right) F\left(W, x, x^{\prime}\right)
$$

To give this expression meaning Hartle and Hawking analytically continue the variables to complex values where the integral is well defined. In particular $w$ and $W$ are continued to negative imaginary values, $-i \omega$ and $-i \Omega$, and the coordinates are continued to a domain where the metric signature is +4 , i.e. $t \rightarrow i \tau$ 
the analytically continued metric being denoted by $\gamma_{\alpha \beta}$. They then notice that $F\left(\Omega, x, x^{\prime}\right)$ satisfies the parabolic partial differential equation:

$$
\frac{\partial F}{\partial \Omega}=-\tilde{\square}^{2} F, \quad \tilde{\square}^{2}=\gamma^{\alpha \beta} \tilde{\nabla}_{\alpha} \tilde{\nabla}_{\beta},
$$

with the boundary condition $F\left(0, x, x^{\prime}\right)=\delta\left(x, x^{\prime}\right)$, while $K\left(x, x^{\prime}\right)$ satisfies:

$$
\left(\square^{2}-m^{2}\right) K\left(x, x^{\prime}\right)=-\delta\left(x, x^{\prime}\right) .
$$

The small $W$ behaviour of $F$, when analytically continued back to real coordinates is found to be:

$$
F\left(W, x, x^{\prime}\right)=\sum_{c} e^{i S_{c}\left(x, x^{\prime}\right) / 4 W} W^{-2}\left[D_{c}\left(x, x^{\prime}\right)+\mathcal{O}\left(w^{-2}\right)\right]
$$

where $D_{c}$ is a function independent of $W$ whose precise form is of no consequence here, and $\sum_{c}$ represents a sum over each class of geodesics connecting $x^{\prime}$ and $x$.

The large $W$ behaviour is also of order $W^{-2}$. Thus the integral (8) always converges at the upper limit, while the lower limit diverges as $W^{-2}$ and is handled by inserting a convergence factor of $e^{-\varepsilon / W}$ with $\varepsilon$ initially finite, and then the limit $\varepsilon \rightarrow 0$ is taken at the end of the calculation:

$$
K\left(x, x^{\prime}\right)=i \int_{0}^{\infty} d W e^{-i m^{2} W-\varepsilon / W} F\left(W, x, x^{\prime}\right) .
$$

Let us now consider $x$ to lie on the complexified horizon defined by $\theta, \phi$ real and $\bar{U} \bar{V}=0$ with $x$ lying in region III + . The analytic properties of $K\left(x, x^{\prime}\right)$ can be deduced from Equations (8), (9) and (10). Since $\gamma_{\alpha \beta}$ is analytic everywhere in the shaded region of Figure $1, F\left(W, x, x^{\prime}\right)$ will also be analytic. Therefore the singularities in $K\left(x, x^{\prime}\right)$ can come only from the $W=0$ end points of the $W$ integration. If the $W$ integration $[0, \infty]$ is divided into two parts $\left[0, W_{0}\right]$ and $\left[W_{0}, \infty\right]$ with $W_{0}$ small, then (10) and (11) imply

$$
K\left(x, x^{\prime}\right)=K_{0}\left(x, x^{\prime}\right)-i \sum_{c} e^{i S_{c}\left(x, x^{\prime}\right) / 4 W_{0}} D_{c}\left(x, x^{\prime}\right) /\left(S_{c}\left(x, x^{\prime}\right)+i \varepsilon\right),
$$

where $K_{0}$ represents the $\left[W_{0}, \infty\right]$ contribution to $K\left(x, x^{\prime}\right)$ and is analytic in $x$. Equation 12 is correct for massless particles and we will now only consider this situation. $K\left(x, x^{\prime}\right)$ has singularities whenever $S_{c}\left(x, x^{\prime}\right)=-i \varepsilon$, i.e. slightly displaced from wherever a null geodesic connects $x^{\prime}$ to $x$.

The expression (12) can be used to continue $K\left(x, x^{\prime}\right)$ off the complexified horizon. First one shows that all null geodesics starting from real values of $x^{\prime}$ intersect the future complexified horizon at real values of $x$ i.e. at real values of $\bar{U}$ and $\bar{V}, \theta, \phi$. Therefore there will be singularities of $K$ on the complex horizon at values slightly displaced from the real values $\bar{U}, \bar{V}$ given by $S\left(x, x^{\prime}\right)=-i \varepsilon$. Then the identical argument of Hartle and Hawking [4] shows that the displacement is such that the singularities occur in the upper half $\bar{V}$ plane on the future horizon and therefore the propagator is analytic in the lower half $\bar{V}$ plane on the future complex horizon.

A similar argument shows that the propagator is analytic in the upper half $\bar{U}$ plane on the complexified past horizon. We shall not repeat the Hartle-Hawking 
argument here, however we will demonstrate that the well known pathologies of Taub-Nut space do not violate the essential prerequisite of the argument that complex null geodesics starting from a real value of $x^{\prime}$ intersect only real values of $x$ on the complexified horizons.

Geodesics in Taub-Nut space have been investigated by Misner and Taub [7]. The necessary equations are obtained by noting that there exist four Killing vectors given by

$$
\begin{aligned}
& \varepsilon_{x}=-\sin \phi \partial_{\theta}-\cos \phi\left(\operatorname{ctg} \theta \partial_{\phi}-\operatorname{cosec} \theta \partial_{\psi}\right) \\
& \varepsilon_{y}=\cos \phi \partial_{\theta}-\sin \phi\left(\operatorname{ctg} \theta \partial_{\phi}-\operatorname{cosec} \theta \partial_{\psi}\right) \\
& \varepsilon_{z}=\partial_{\phi} \\
& \eta=-\partial_{\psi},
\end{aligned}
$$

and hence there exist four constants of the motion:

$$
\begin{aligned}
& p_{a}=v \cdot \xi_{a} \quad a=1,2,3 \\
& p_{\|}=-v \cdot \eta,
\end{aligned}
$$

in addition to the constant relation for null geodesics:

$$
g_{\mu \nu} \frac{d x^{\mu}}{d \lambda} \frac{d x^{\nu}}{d \lambda}=0, \quad \lambda=\text { an affine parameter }
$$

The orientation of the coordinate axis's can be chosen such that $p_{x}=p_{y}=0$ and $p_{z}=p \geqq 0$, which imply $p \cos \theta=p_{\|}$. This last condition is the analogue of the $\theta=\pi / 2$ condition of the spherically symmetric situation of Hartle and Hawking. The Equations $(14 a, b)$ reduce to the following when the metric is the extended one defined by Equation (2) that covers the future horizon: of region III + :

$$
\begin{aligned}
& \left(t^{2}+l^{2}\right) \frac{d \phi}{d \lambda}=p \\
& 2 l U(t) \frac{d v}{d \lambda}+\frac{2 l U(t) \cos \theta p}{t^{2}+l^{2}}-R(t)=p_{\|} / 2 l
\end{aligned}
$$

where:

$$
p_{\perp}=p^{2}-p_{\|}^{2} \quad \text { and } \quad R(t)=\left\{\left(\frac{p_{\|}^{2}}{2 l}\right)+\frac{U(t) p_{\perp}^{2}}{t^{2}+l^{2}}\right\}^{1 / 2} .
$$

Then the following expressions result from the null conditions (15)

$$
\begin{aligned}
& \phi-\phi^{\prime}=\int_{t^{\prime}}^{t-} d t p /\left(t^{2}+l^{2}\right) R(t) \\
& v-v^{\prime}=\int_{t^{\prime}}^{t_{-}} \frac{1}{2 l U(t)}\left[1+\frac{p_{\|}}{2 l R(t)}-\frac{2 l U p_{\|}}{\left(t^{2}+l^{2}\right) R(t)}\right] .
\end{aligned}
$$

Since $\theta$ must be real (since $\theta=$ constant $=\theta^{\prime}$, the real value of $\theta$ at the real point $x^{\prime}$ ) the relation (above) $p \cos \theta=p_{\|}$implies that $p_{\|}$is also real, since $p$ can be 


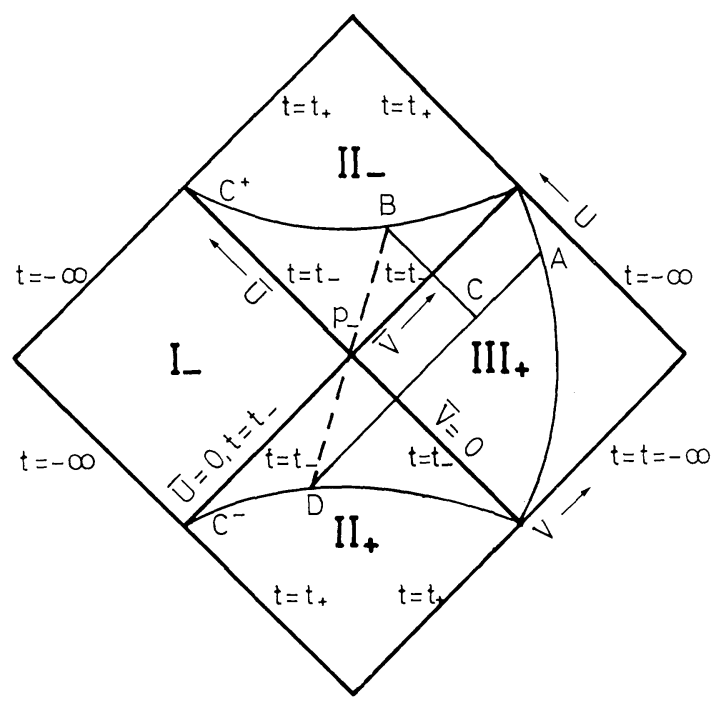

Fig. 2. Penrose diagram of the relevant region of Taub-Nut space. $C^{+}$is a space like surface connecting the intersections of the past and future horizons of region $\mathrm{II}-. \mathrm{C}^{-}$is the reflection of $C^{+}$through the (deleted) point $p_{\ldots}$. An observer travels on the (closed) timelike curve $t=$ const. $D$ is the reflection of point $B$ through the (deleted) point $p_{-}$

chosen to be real by utilising the multiplicative arbitrariness of the affine parameter $\lambda$. An elementary analysis of the integrand (17) then shows that if $p_{\|}>f\left(m, l, p_{\perp}\right)$, where $f\left(m, l, p_{\perp}\right)$ is the absolute value of the minimum value of $U(t) p_{\perp}^{2} /\left(t^{2}+l^{2}\right)$, then $\phi$ will be real as required. However from Equation (18) this is also seen to be the necessary condition that $v$ be real. Hence complex null geodesics starting from real values $v^{\prime} t^{\prime} \theta^{\prime} \phi^{\prime}$ intersect the future horizon at real values $v t \theta \phi$. Thus the Hartle-Hawking argument deriving the result that the singularities of $K\left(x, x^{\prime}\right)$ occur in the upper half $\bar{V}$ plane and the lower half $\bar{U}$ plane is valid for Taub-Nut space.

We can now continue $K\left(x, x^{\prime}\right)$ off the complexified horizon. For definiteness consider $x^{\prime}$ in region III + and $x$ in region II - . The portion of the future horizon with $\bar{V} \geqq 0$ together with the portion of the past horizon with $\bar{U}>0$ are a complete characteristic Cauchy surface for region II - . The propagator in region IIis therefore determined by data on this Cauchy surface which is just the values of the propagator on the relevant parts of the horizon. Write the complex $\psi$ as $\psi=\kappa+i \sigma$ so that $2,3,4 \mathrm{a}, 4 \mathrm{~b}$ imply

$$
\begin{aligned}
& \bar{U}=|\bar{U}| e^{-i \kappa-\sigma} \\
& \bar{V}=|\bar{V}| e^{i \kappa-\sigma} .
\end{aligned}
$$

Then the problem of determing the propagator at a certain complex value of $\psi$ is equivalent to solving the hyperbolic Equation (9b) in the real coordinates $|\bar{U}|$ and $|\bar{V}|$. This reduces to the characteristic initial value problem with Cauchy data provided along the portions of the horizons mentioned above. The data is regular so long as $-\pi / \kappa_{-}<\sigma<0$. Since the metric is analytic in the region of interest, 
and the characteristic Cauchy data is regular so long as $-\pi / \kappa_{-}<\sigma<0$ the propagator, $K\left(x, x^{\prime}\right)$, with $x^{\prime}$ in region III + , and $x$ at fixed $t \theta \phi$ in the region $\bar{U}>0$, $\vec{V}>0$ will be analytic in $\psi$ in a width of $\pi / \kappa_{-}$below the real axis. The proviso should be added that no new Cauchy data enter from the delected points $p_{-}, p_{+}$.

This result is sufficient to derive the thermal spectrum of radiation seem by an observer moving on a (closed) time-like line of constant $t=t^{\prime}$ in the NUT region III + . Consider Figure 2. Let us impose the condition that no particles enter region III + across its past horizon or from $t=-\infty$. Then any particle in a mode with energy $+E$ detected by an observer at the point $A$ in Figure 2 must have come from region II - . A typical worldline of such a particle is the path $B C A$. In the usual Feynman picture such a particle propagating backward from the region II - is interpreted as the creation of a pair at point $C$, one particle of which crosses the future horizon the other propagating to $A$. Any such particle propagating backwards from region II - must cross an arbitrary space like surface connecting $t=t_{-}$and $t=t_{+}$, labelled as the surface $C_{+}$in Figure 2 . We have said that this particle is detected at $A$ with positive energy $E$ with respect to the time coordinate $\psi^{\prime}$; so that it is in a mode $e^{-i E \psi^{\prime}} f_{j}(\vec{R})$ at $t=t^{\prime}$, where $\vec{R}$ refers to the coordinates $t^{\prime}, \theta^{\prime}, \phi^{\prime}$. By the time translation invariance of the Taub-Nut solution this mode must have had the form $e^{-i E \psi} h_{j}\left(\vec{R}^{\prime}\right)$ when it crossed the surface $C_{+}$.

Therefore, the amplitude that a particle is detected in a mode $f_{j}\left(x^{\prime}\right)$ having originated in a mode $h_{j}(x)$ on the surface $C^{+}$is:

$-\int d \sigma^{\mu}\left(x^{\prime}\right) \int d \sigma^{\nu}(x) \bar{f}_{i}\left(x^{\prime}\right) \overleftrightarrow{\partial_{\mu}} K\left(x, x^{\prime}\right) \overleftrightarrow{\partial_{\nu}} h_{j}(x)$

where, with the given boundary conditions, the integral over $x$ extends over the surface $C^{+}$and the $x^{\prime}$ integral is over the surface $t=t^{\prime}$. The notation $a \overleftrightarrow{\partial u} b$ means $a b,{ }_{u}-b a,{ }_{u}$. The crucial information about the emission process is contained in the part of the integral (20) that has the form:

$$
\mathscr{E}_{E}\left(\vec{R}^{\prime}, \vec{R}\right)=\int_{0}^{4 \pi} d \psi e^{-i E \psi} K\left(0, \vec{R}^{\prime} ; \psi, \vec{R}\right) .
$$

This is the amplitude for the emission of a particle with energy $E$ detected at $\left(0, \vec{R}^{\prime}\right)$. Since $K\left(\psi, \vec{R}, 0 \vec{R}^{\prime}\right)$ is analytic in a strip of width $\pi / \kappa_{-}$below the real $\psi$ axis we distort the contour of the $\psi$ integration in Equation (21) downward by $-i \pi / \kappa_{-}$. However, by Equation (19) this is equivalent to reflecting the $\bar{U}, \bar{V}$ coordinates through the point $\varrho_{-}$of Figure 2 . Thus the translated integral:

$$
\mathscr{E}_{E}\left(x, x^{\prime}\right)=e^{-\pi E / \kappa}-\int_{0}^{4 \pi} d \psi e^{-i E \psi} K\left(\psi-i \pi / \kappa_{-}, \vec{R} ; 0, \vec{R}^{\prime}\right)
$$

can be interpreted as the component with energy $E$ of the amplitude to propagate from the surface $C^{-}$in Figure 2, which is the reflection of $C^{+}$through $p_{-}$, to a point $\left(0, \vec{R}^{\prime}\right)$ in region III + . However by time reversal invariance the modulus squared of this amplitude is equal to the modulus squared of the amplitude to absorb a particle which starts at $\left(0, \vec{R}^{\prime}\right)$ with energy $E$ and arrives at $C_{+}$. We therefore have the relation

$$
\left(\begin{array}{l}
\text { Probability of emission } \\
\text { of particle with energy } E
\end{array}\right)=e^{-2 \pi E / \kappa}-\left(\begin{array}{l}
\text { Probability of absorption of } \\
\text { particle with energy } E
\end{array}\right)
$$


which is the fundamental connection between emission and absorption indicating a thermal spectrum characterized by a temperature of $T=\kappa_{-} / 2 \pi$. Since the observer at constant $t^{\prime}$ is travelling on a closed timelike line the lowest frequency he can detect is $1 / 4 \pi$ where $4 \pi$ is his fundamental period. Therefore the thermal spectrum is truncated at a lower frequency limit of $1 / 4 \pi$, and is discrete at integer multiplies of $1 / 4 \pi$.

The back reaction of the particle production has a drastic effect on Taub-Nut space. Misner [8] has shown that Taub Nut space is unstable toward matter perturbations and that a curvature singularity develops in Taub-Nut space upon the introduction of a perfect fluid matter perturbation. We have shown above that it is not necessary to put in this perturbation explicitly since Taub-Nut space has a built in instability caused by the spontaneous creation of particles from the quantum mechanical vacuum.

It is realized that Taub-Nut space is a very unphysical spacetime and hence is not the best arena in which to attempt physical explanations of the "Hawking process". In particular, the use of an observer moving on a closed time-like line grates against one's physical sensibilities. However this ubiquity of thermal spectra is interesting and ties in with the thermodynamic idea of thermal spectra being associated with arbitrary event horizons (not just black hole horizons) basically through the Kirchoff principle of a perfect absorber necessarily being also a perfect emitter. A less unusual spacetime is considered in the next section.

\section{Type VIII Taub-Nut Space: The Global Properties}

When the Nut continuation [5] of Taub space [7] was discovered it was regarded as a generalization of the Schwarzschild solutions which had singularities on the axis. Misner [8] showed however that this singularity could be removed if the $t=$ constant sections of the space had topology $S^{3}$ instead of $R \times S^{2}$. The simplest way to see that the coordinate $\psi$ must be "wrapped up", yielding a spacetime of topology $R \times S^{3}$ is to consider the line element (1) written in the orthonormal basis:

$$
\begin{aligned}
& \omega^{0}=U(t)^{-1 / 2} d t \\
& \omega^{\prime}=U^{1 / 2}\left[d \tilde{\psi}+4 l \sin ^{2} \frac{\theta}{2} d \phi\right] \\
& \omega^{2}=\left(t^{2}+l^{2}\right)^{1 / 2} d \theta \\
& \omega^{3}=\left(t^{2}+l^{2}\right)^{1 / 2} \sin \theta d \phi \\
& d s^{2}=\eta_{u v} \omega^{u} \omega^{v}
\end{aligned}
$$

Thus:

$$
d \bar{\psi}=U^{-1 / 2} \omega^{1}-2 l\left(t^{2}+l^{2}\right)^{-1 / 2} \tan \frac{\theta}{2} \omega^{3}
$$

so

$$
(\operatorname{grad} \bar{\psi})^{2}=U^{-1}+(2 l)^{2}\left(t^{2}+l^{2}\right)^{-1} \tan \frac{\theta}{2}
$$




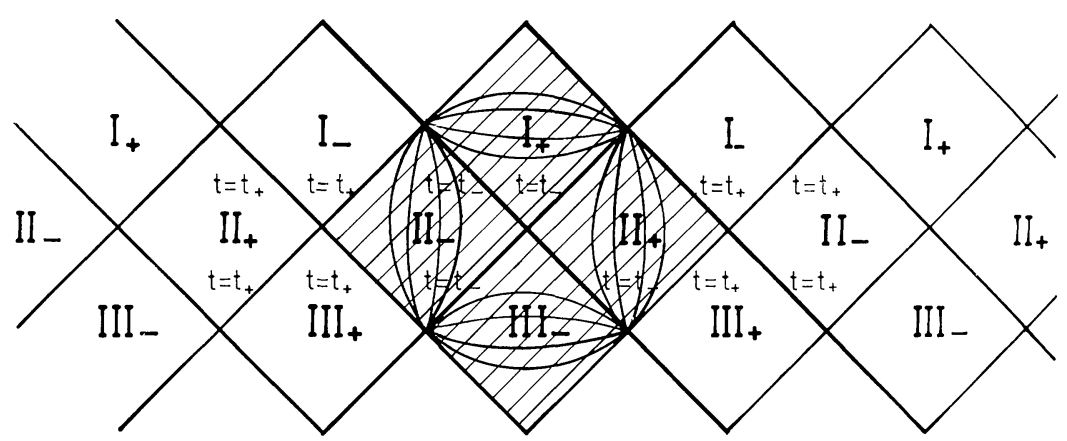

Fig. 3. The Penrose diagram of the maximal extension of Type VIII Taub-Nut space

and therefore the coordinate $\bar{\psi}$ is regular only over $\theta \in[0, \pi)$. A new coordinate $\overline{\bar{\psi}}$, defined by:

$$
\overline{\bar{\psi}}=\bar{\psi}+4 l \varphi
$$

is easily seen to be regular over the range $\theta \in(0, \pi]$. Thus $\bar{\psi}$ can be used in the coordinate patch $\theta \in[0, \pi)$, while $\overline{\bar{\psi}}$ can be used in $\theta \in(0, \pi]$. However the periodicity of $\varphi$ must be respected in the overlap region $\theta \in(0, \pi)$ so the $\bar{\psi}, \bar{\psi}$ coordinates must be periodic with period $8 \pi l$. The coordinate transformation:

$$
\bar{\psi}=-2 l(\phi+\psi)
$$

then introduces the time coordinate $\psi$ of period $4 \pi$ used in Equation (1).

The observation has been made by Siklos [3] that replacing $\theta$ by $i \theta$ turns the trigonometric functions singular at $\theta=\pi$ into hyperbolic functions regular over $\theta \in(-\infty, \infty)$. This replacement results in a different vacuum solution of Einstein's equations with signature ---+ and is Bianchi-type VIII. The original TaubNut space is Bianchi type IX. Since the axis singularity is removed it is no longer necessary to wrap up the $\psi$ coordinate which leaves only one periodic coordinate $\varphi$. Since $\cos \theta$ is replaced by $\cosh \theta$ and $\sin ^{2} \theta$ by $-\sinh ^{2} \theta$ the line element can be written:

$$
d s^{2}=-U^{-1} d t^{2}+(2 l)^{2} U(d \psi+\cosh \theta d \phi)^{2}-\left(t^{2}+l^{2}\right)\left(d \theta^{2}+\sinh ^{2} \theta d \varphi^{2}\right)
$$

where:

$$
\begin{aligned}
& t \in(-\infty, \infty) \\
& \theta \in(-\infty, \infty) \\
& \psi \in(-\infty, \infty) \\
& \varphi \in[0,2 \pi] .
\end{aligned}
$$

The global extension of (29) can be made in an analogous fashion to the extensions of Taub-Nut space discussed in Section 1. Since $\psi$ is no longer periodic the extended spacetime is a Hausdorff manifold that contains the points $p_{ \pm}$ which were deleted in Taub-Nut space in order to make that extension a manifold. 
The Penrose diagram of the global extension of (29) is shown in Figure 3. In the Type VIII Taub-Nut spacetime, above, the lines $t, \theta, \varphi=$ constant, $\psi=\psi(\lambda)$ are no longer closed time like lines in regions $\mathrm{I} \pm, \mathrm{III} \pm$ as they were in the original Taub-Nut space. However there do exist closed timelike lines even in the unwrapped spacetime e.g. $t, \psi=$ constant; $\theta=0 ; \varphi=\varphi(\lambda)$. The key feature of this spacetime is that an observer moving on a $t, \theta, \phi=$ const., $\psi=\psi(\lambda)$ world line in region $\mathrm{II}+$ is not travelling on a closed time like line and we show in Section 4 that this observer would detect a thermal spectrum of radiation.

It seems that Type VIII Taub-Nut spacetime is stable toward the introduction of matter perturbations by considering the physical arguments used by Misner [7] to show that Taub-Nut spacetime is unstable. We will not repeat these arguments here but merely note that each argument for instability depends crucially on the periodicity of $\psi$ in the Taub-Nut metric. By analytically continuing and unwrapping $\psi$ we remove this instability. It therefore becomes possible to discuss the effect of the back reaction of the thermal radiation in slightly more detail than is possible for Type IX Taub-Nut spacetime.

\section{The Hartle-Hawking Method: Type VIII Taub-Nut Space}

The Hartle Hawking method has been reviewed and applied to Taub-Nut space in Section 2. In that section it was shown that an observer moving on a (closed) time like line of $t, \theta, \phi=$ constant would detect a truncated and discrete thermal spectrum of radiation. The application of this method to Type VIII Taub-Nut space proceeds in a virtually identical manner. The analogue of the points $B D C A$ and the surfaces $C_{ \pm}$are shown in Figure 4. The observer is chosen to move on a (non closed) time like world line of $t, \theta, \phi=$ constant in region $\mathrm{II}+$ and can now detect radiation coming from the two horizons $t=t_{+}$and $t=t_{-}$characterized by the different surface gravities $\kappa_{+}$and $\kappa_{-}$.

The existence of two horizons with two different surface gravities creates a problem. One can introduce two Kruskal type coordinates patches, completely analogous to $U_{+} V_{+}, U_{-} V_{-}$of Equations (4), that cover the $t=t_{+}, t=t_{-}$horizons separately. In the overlap region the coordinates of one patch will be real analytic functions of the other. However, branch cuts will arise when $U_{ \pm}$and $V_{ \pm}$are complexified, since by Equations (4)

$$
\left(\frac{-\bar{V}_{-}}{\bar{U}}\right)=\left(-\bar{V}_{+} / \bar{U}_{+}\right)^{\kappa-/ \kappa_{+}}
$$

and a branch cut exists unless $\kappa_{+}=\kappa_{-}$.

We avoid this problem by inserting a perfectly reflecting wall between the horizons and impose reflecting boundary conditions on $K\left(x, x^{\prime}\right)$ at the wall. Then an observer on one side of the wall would see a thermal spectrum characterised by the surface gravity of one horizon, and an observer on the opposite side would see a thermal spectrum characterized by the surface gravity of the other horizon. Presumably if the walls were removed the observer would detect a mixture of radiation.

Since Type VIII Taub-Nut space seems to be stable under the introduction of matter perturbations one can say slightly more about the back reaction of the radiation than was possible in the Type IX space. Consider two slightly different 


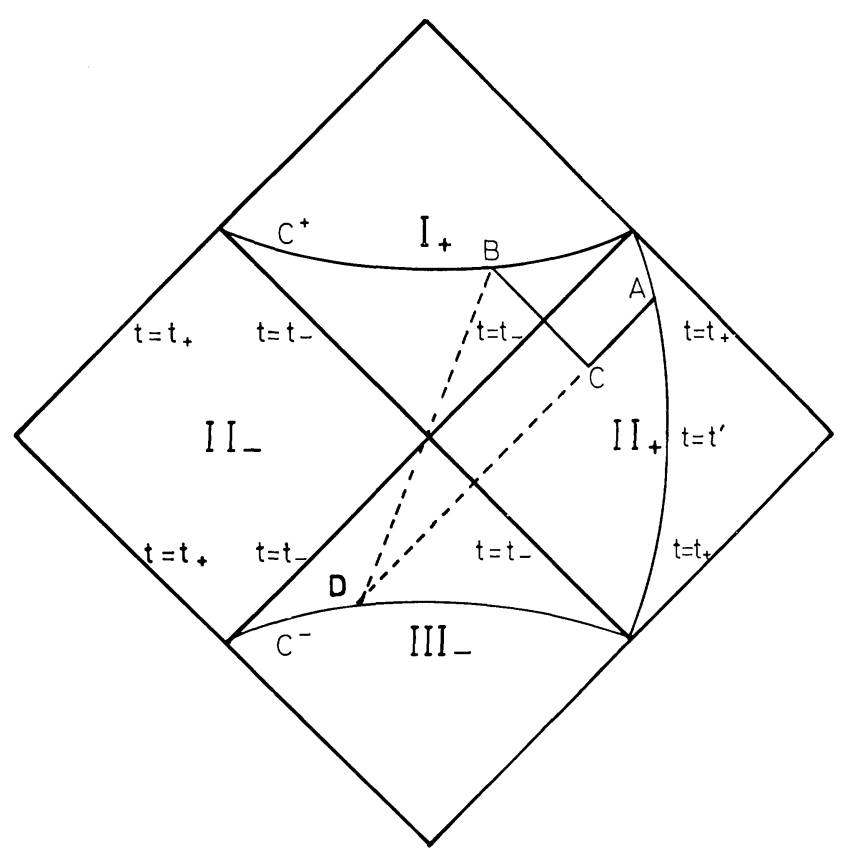

Fig. 4. The relevant section of the Penrose diagram of Type VIII Taub-Nut space. The surfaces $C_{+}$, $C_{-}$and points $B D C A$ are the analogue of the points in Figure 2

type VIII Taub-Nut solutions that contain matter between the event horizons $t=t_{-}$and $t=t_{+}$. The difference will be caused by the absorption of a small amount of thermal radiation by the observer. Denote the difference of the stress energy tensors $\mathrm{T}_{a b}$ of the two solutions as $\delta \mathrm{T}_{a b}$ and denote the difference of the areas of the stwo event horizons as $\delta A_{+}$and $\delta A_{-}$. Then the Gibbons and Hawking generalization [1] of the first law of black hole mechanics as applied to our situation states

$$
\int \delta T_{a b} K^{a} d \Sigma^{b}=-\frac{\kappa_{+} \delta A_{+}}{8 \pi}-\frac{\kappa_{-} \delta A_{-}}{8 \pi}
$$

where

$\delta A_{+}=$difference in area of the $t=t_{+}$event horizons of the two solutions,

$\delta A_{-}=$difference in area of the $t=t_{-}$event horizons of the two solutions,

$\kappa_{ \pm}=$surface gravities of $t=t_{ \pm}$horizons respectively

$\delta \mathrm{T}_{a b}=$ difference in the energy momentum tensor between the horizons in the two solutions.

If an observer absorbs a small amount of energy of the thermal radiation then the change in $\mathrm{T}_{a b}$ is positive, indicating that the area of the relevant horizons decreases from their original infinite value to another infinite value. However since a solution that is asymptotically Type VIII Taub-Nut space containing a mass moving 
on a time like line is not known, it is impossible to say how the surface gravities $\kappa_{ \pm}$ will change.

\section{Conclusions}

We have shown that in one highly unphysical and pathological spacetime, TaubNut space, the Hartle-Hawking path integral method originally used to derive black hole radiance is applicable and predicts a thermal spectrum of radiation for a suitable observer. Taub-Nut space can be analytically continued and unwrapped [3] to yield a more physical anisotropic cosmological model, and a suitable observer will also see a thermal spectrum. The elegance of the calculational method prompts the conjecture that in any spacetime with crossed horizons and vertical symmetry a suitable observer would detect a thermal spectrum. This ubiquity of thermal spectra lends support to the ideas advocated by Hawking [9] that a deep connection exists between thermodynamics and gravitation (in particular the existence of event horizons). We stress that these results are necessarily observer dependent and emphasize that whether an observer sees a thermal spectrum or not depends on his state of motion. This can be most clearly seen in the anti-de Sitter [6] and Bertotti [10] universes which will be discussed in a subsequent paper.

Acknowledgements. I am grateful to S.W.Hawking and G.W. Gibbons for helpful discussions.

\section{References}

1. Gibbons, S.W., Hawking, S. W.: Cosmological event horizons, thermodynamics and particle creation. DAMTP, University of Cambridge, preprint

2. Hartle, J., Hawking, S. W.: Path integral derivation of black hole radiance. Cal. Tech. Orange Aid preprint

3. To be published

4. Taub, A.: Ann. Math. 53, 472 (1951)

5. Newman, E., Tamburino,L., Unti,T.: JMP 4, 915 (1963)

6. Hawking, S.W., Ellis, G.F.R.: The large scale structure of space time. Cambridge: Cambridge University Press 1973

7. Misner, C., Taub, A.: JETP 28, 122 (1968)

8. Misner, C.: JMP 4, 924 (1963)

9. Fundamental breakdown of Physics. Cal. Tech. Orange Aid preprint

10. Bertotti, B.: Phys. Rev. 116, 1331 (1959)

Communicated by R. Geroch

Received June 1, 1976 
